\title{
Universality of Fragmentation in the Schrödinger Dynamics of Bosonic Josephson Junctions
}

\author{
Kaspar Sakmann ${ }^{1}$, Alexej I. Streltsov ${ }^{2}$, Ofir. E. Alon ${ }^{3}$, and Lorenz S. Cederbaum ${ }^{2}$ \\ 1 Department of Physics, Stanford University, Stanford, California 94305, USA \\ 2 Theoretische Chemie, Physikalisch-Chemisches Institut, Universität Heidelberg, \\ Im Neuenheimer Feld 229, D-69120 Heidelberg, Germany and \\ 3 Department of Physics, University of Haifa at Oranim, Tivon 36006, Israel
}

(Dated: November 5, 2018)

\begin{abstract}
The many-body Schrödinger dynamics of a one-dimensional bosonic Josephson junction is investigated for up to ten thousand bosons and long times. The initial states are fully condensed and the interaction strength is weak. We report on a universal fragmentation dynamics on the many-body level: systems consisting of different numbers of particles fragment to the same value at constant mean-field interaction strength. The phenomenon manifests itself in observables such as the correlation functions of the system. We explain this universal fragmentation dynamics analytically based on the Bose-Hubbard model. We thereby show that the extent to which many-body effects become important at later times depends crucially on the initial state. Even for arbitrarily large particle numbers and arbitrarily weak interaction strength the dynamics is many-body in nature and the fragmentation universal. There is no weakly interacting limit where the Gross-Pitaevskii mean-field is valid for long times.

PACS numbers: 05.60.Gg,03.75.Kk,05.30.Jp,03.65.-w
\end{abstract}

\section{INTRODUCTION}

Over the last few years ultracold bosons in doublewell potentials have allowed the exploration of quantum many-body dynamics in a highly controllable manner. Among the phenomena predicted or observed are tunneling and self-trapping [1 [5], Josephson oscillations [2 5], collapse and revival sequences [1], squeezing [6], and matter wave interferometry [7], to name a few. While tunneling, self-trapping and Josephson oscillations have explanations on the mean-field level, other phenomena, e.g., collapse and revival sequences and fragmentation dynamics require many-body treatments such as the Bose-Hubbard model [1] or even the full many-body Schrödinger equation [8, 9]. Of particular relevance to this work is condensate fragmentation 10, 11], i.e. the occurrence of several macroscopically occupied quantum states as opposed to conventional Bose-Einstein condensation with just one macroscopically occupied state [12]. Condensate fragmentation has been predicted to occur [8, 13 16] and observed in the ground state [17], see also 6] in this context. It is a many-boson phenomenon due to interparticle interactions. Generally, stronger interactions lead to more fragmentation, but the question to what extent a condensate fragments usually depends intricately on the number of bosons and the interaction strength.

In bosonic Josephson junctions self-trapping - the inhibition of tunneling due to interparticle interactions occurs from a critical value of the interaction strength onwards within two-mode Gross-Pitaevskii mean-field theory [1, 2]. This critical value provides a reference point for the importance of interactions in the system. Two-mode GP mean-field theory was successfully applied in the description of bosonic Josephson junction experiments at short time scales [3 [5]. However, for longer times the dynamics can leave the realm of mean-field theory and make a many-body description necessary, see, e.g., Refs. $[1,8,18,33]$.

This paper contains the following main results. Firstly, we investigate the long-time many-body dynamics of large Bose-Einstein condensates (BECs) of up to ten thousand bosons. To this end we solve the timedependent many-body Schrödinger equation numerically for initially fully condensed BECs in a one-dimensional bosonic Josephson junction. We consider interaction strengths that are so weak that self-trapping cannot occur. We report on the existence of a universal manybody fragmentation dynamics in bosonic Josephson junctions: systems consisting of different numbers of bosons all fragment to the same value at fixed mean-field interaction strength. The occurrence of such a universal many-body dynamics is unexpected and is shown here explicitly based on the many-body Schrödinger equation for up to 10000 bosons. The phenomenon manifests itself in observables, such as the correlation functions.

Secondly, using the Bose-Hubbard (BH) model we explain analytically how the universal fragmentation dynamics depends on the initial state and its occurrence for arbitrarily large numbers of particles and arbitrarily weak mean-field interaction strength. We show that for a whole class of condensed initial states the analytical calculations allow for precise predictions about the fragmentation of the BEC after the collapse of the density oscillations and thus about the extent to which the many-body dynamics remains within the realm of meanfield. We thereby show that there is no limit for which GP theory remains valid at long times. 


\section{THEORETICAL FRAMEWORK}

In this section we introduce the theoretical methods and quantities used in this work to explore the dynamics of a one-dimensional bosonic Josephson junction. In the following we suppress the time argument whenever possible.

\section{A. Many-body Hamiltonian, wavefunction and parameters}

It is convenient to use dimensionless units defined by dividing the Hamiltonian by $\frac{\hbar^{2}}{m L^{2}}$, where $m$ is the mass of a boson and $L$ is a length scale. The full many-body Hamiltonian then reads

$$
H=\sum_{i=1}^{N} h\left(x_{i}\right)+\sum_{i<j} W\left(x_{i}-x_{j}\right)
$$

where $h(x)=-\frac{1}{2} \frac{\partial^{2}}{\partial x^{2}}+V(x)$ with a trapping potential $V(x)$ and an interparticle interaction potential $W(x-$ $\left.x^{\prime}\right)=\lambda_{0} \delta\left(x-x^{\prime}\right)$. For the potential $V(x)$ we choose a double-well constructed by connecting two harmonic potentials

$$
V_{ \pm}(x)=\frac{1}{2}(x \pm 2)^{2}
$$

with a natural cubic spline at $|x|=0.5$. This uniquely defines the external potential $V(x)$. The even-symmetry ground state $\phi_{g}$ and odd-symmetry excited state $\phi_{u}$ of the one-particle double-well Hamiltonian $h(x)$ allow to define several commonly used parameters. First two leftand right-localized Wannier functions

$$
\phi_{L, R}=\frac{1}{\sqrt{2}}\left(\phi_{g} \pm \phi_{u}\right)
$$

are constructed. This defines the hopping parameter

$$
J=-\left\langle\phi_{L}|h| \phi_{R}\right\rangle=2.2334 \times 10^{-2},
$$

as well as the interaction parameter

$$
U=\lambda_{0} \int d x\left|\phi_{L}(x)\right|^{4}
$$

and the single particle tunneling time scale $t_{\text {Rabi }}=$ $\pi / J=140.66$.

In second quantization the Hamiltonian (1) of the many-boson system reads

$\hat{H}=\int d x \hat{\Psi}^{\dagger}(x) h(x) \hat{\Psi}(x)+\frac{\lambda_{0}}{2} \int d x \hat{\Psi}^{\dagger}(x) \hat{\Psi}^{\dagger}(x) \hat{\Psi}(x) \hat{\Psi}(x)$.

The theoretical approaches employed in this work, namely the Bose-Hubbard model (dimer) and the multiconfigurational time-dependent Hartree for bosons
(MCTDHB) method [20] can be derived from different truncations of the field operator

$$
\hat{\Psi}(x)=\sum_{j} \hat{b}_{j} \phi_{j}(x) .
$$

Here, $\left\{\phi_{j}(x)\right\}$ denotes a complete orthonormal set of orbitals, $\hat{b}_{j}$ annihilates a boson in $\phi_{j}(x)$ and $\left[\hat{b}_{i}, \hat{b}_{j}^{\dagger}\right]=\delta_{i j}$. In practice, the infinite set $\left\{\phi_{j}(x)\right\}$ is truncated to a finite number $M$. Associated with a truncation of the field operator to $M$ terms is an expansion of the $N$-boson wavefunction

$$
|\Psi\rangle=\sum_{\vec{n}} C_{\vec{n}}|\vec{n}\rangle
$$

with expansion coefficients $C_{\vec{n}}$ and basis vectors

$$
|\vec{n}\rangle=\frac{\hat{b}_{1}^{\dagger n_{1}} \hat{b}_{2}^{\dagger n_{2}} \cdots \hat{b}_{M}^{\dagger n_{M}}}{\sqrt{n_{1} ! n_{2} ! \cdots n_{M} !}}|0\rangle .
$$

Here, $n_{1}+\cdots+n_{M}=N$ is implied. There are $\left(\begin{array}{c}N+M-1 \\ N\end{array}\right)$ expansion coefficients $C_{\vec{n}}$.

\section{B. Bose-Einstein condensation and fragmentation}

For a given many-boson wavefunction $|\Psi\rangle$ all one-body observables can be computed from the first-order reduced density matrix

$$
\begin{aligned}
\rho^{(1)}\left(x \mid x^{\prime}\right) & =\left\langle\Psi\left|\hat{\Psi}^{\dagger}\left(x^{\prime}\right) \hat{\Psi}(x)\right| \Psi\right\rangle \\
& =\sum_{i j} \rho_{i j} \phi_{j}(x) \phi_{i}^{*}\left(x^{\prime}\right) \\
& =\sum_{i} n_{i}^{(1)} \alpha_{i}^{(1)}(x) \alpha_{i}^{(1) *}\left(x^{\prime}\right),
\end{aligned}
$$

where $\rho_{i j}=\left\langle\Psi\left|\hat{b}_{i}^{\dagger} \hat{b}_{j}\right| \Psi\right\rangle$ and the eigenfunctions $\alpha_{i}^{(1)}(x)$ and eigenvalues $n_{1}^{(1)} \geq n_{2}^{(1)} \geq \ldots$ are known as natural orbitals and natural occupations, respectively. The natural occupations satisfy $\sum_{i} n_{i}^{(1)}=N$ and the singleparticle density is given by

$$
\rho(x)=\rho^{(1)}(x \mid x) .
$$

Bose-Einstein condensation is defined as follows: if only one nonzero eigenvalue $n_{1}^{(1)}=\mathcal{O}(N)$ exists, the system is condensed [12]. If there is more than one such eigenvalue, the BEC is said to be fragmented [10, 13 15, 27].

For our purposes it is convenient to quantify fragmentation using the quantity

$$
f=\frac{1}{N} \sum_{i>1} n_{i}^{(1)}
$$

which we refer to as the fragmentation for simplicity. For fully condensed states, i.e. $n_{1}^{(1)}=N$, one finds $f=0$, 
whereas $0 \leq f<1$ in general. Truncating the field operator to $M$ terms leads to an $M$ by $M$ first-order reduced density matrix $\rho_{i j}$, implying $f \leq \frac{M-1}{M}$. Fragmentation manifests itself in correlation functions and fringe visibilities [27]. Several experiments have measured the effects of fragmentation, see for instance [6, 17, 30].

In the strict sense the definition of BEC given above makes use of the thermodynamic limit [12]. It is nevertheless common practice to apply it also to finite, trapped systems, such as those realized in experiments 11].

In the thermodynamic limit Bose-Einstein condensation has been interpreted as the largest eigenvalue of the one-particle reduced density matrix, $n_{1}^{(1)}$ being an extensive rather than an intensive quantity [12]. The fragmentation $f$ as defined in Eq. (12) would then be an intensive quantity. This interpretation does not hold for trapped, finite systems: the ground states of some trapped, interacting BECs of finite size are known to be fragmented, i.e. $f>0$, see, e.g., [6, 13 17]. However, it can be rigorously proven that $f=0$ for the ground states of trapped BECs at any finite mean-field interaction strength $\lambda_{0}(N-1)$ in the limit $N \rightarrow \infty$ [32]. Thus, fragmentation is dependent on $N$ and cannot be interpreted as an intensive quantity in trapped finite systems. In nonequilibrium dynamics problems, which we discuss in this work, the fragmentation generally does not only change with the particle number and mean-field interaction strength, but also in time.

\section{Probability in the left well and correlation functions}

In the dynamics of Bose-Einstein condensates in double-well potentials it is instructive to consider the integral over the probability density in the left half of the double-well potential

$$
p_{L}(t)=\frac{1}{N} \int_{-\infty}^{0} \rho(x ; t) d x .
$$

The probability in the right half of space then follows from normalization $p_{R}(t)=1-p_{L}(t)$.

Correlation functions can be derived from the reduced density matrices of all orders, e.g. the single-particle momentum distribution and the single-particle density derive from the first-order reduced density matrix, Eq (10). It is possible to answer the question whether a condensate is fragmented or not from the simultaneous measurement of the single-particle density and momentum distribution.

However, second-order correlation functions are particularly appealing because they provide the answer to this question through the measurement of a single quantity. Second-order correlation functions of Bose-Einstein condensate have been measured in many recent experiments see, e.g., Refs. [29 31].
The momentum correlation function is defined as

$$
g^{(2)}\left(k, k^{\prime}\right)=\frac{\rho\left(k, k^{\prime}\right)}{\rho(k) \rho\left(k^{\prime}\right)},
$$

where

$$
\rho(k)=\left\langle\Psi\left|\hat{\Psi}^{\dagger}(k) \hat{\Psi}(k)\right| \Psi\right\rangle
$$

is the single-particle and

$$
\rho\left(k, k^{\prime}\right)=\left\langle\Psi\left|\hat{\Psi}^{\dagger}(k) \hat{\Psi}^{\dagger}\left(k^{\prime}\right) \hat{\Psi}\left(k^{\prime}\right) \hat{\Psi}(k)\right| \Psi\right\rangle
$$

the two-particle momentum distribution. For fully condensed states the momentum correlation function is constant, $g^{(2)}\left(k, k^{\prime}\right)=1-1 / N$, indicating that there are no correlations between momenta, see, e.g., [27, 28]. The presence of momentum correlations in BECs therefore indicates unambiguously the presence of fragmentation.

\section{Bose-Hubbard model}

A popular many-body model for the description of bosons in a symmetric double-well is the $\mathrm{BH}$ model for two sites. The BH Hamiltonian for a double-well potential can be obtained explicitly from Eq. (6) by restricting the field operator to a sum of only two terms

$$
\hat{\Psi}(x)=\hat{b}_{L} \phi_{L}(x)+\hat{b}_{R} \phi_{R}(x) .
$$

using the left- and right-localized Wannier functions, Eq. (3).

By substituting Eq. (17) into the many-body Hamiltonian (6), neglecting the off-diagonal interaction terms (e.g., $\hat{b}_{L}^{\dagger} \hat{b}_{L}^{\dagger} \hat{b}_{L} \hat{b}_{R}$ ), and by eliminating the diagonal onebody terms (e.g., $\left.\hat{b}_{R}^{\dagger} \hat{b}_{R}\right)$, one readily arrives at the BoseHubbard Hamiltonian

$\hat{H}_{B H}=-J\left(\hat{b}_{L}^{\dagger} \hat{b}_{R}+\hat{b}_{R}^{\dagger} \hat{b}_{L}\right)+\frac{U}{2}\left(\hat{b}_{L}^{\dagger} \hat{b}_{L}^{\dagger} \hat{b}_{L} \hat{b}_{L}+\hat{b}_{R}^{\dagger} \hat{b}_{R}^{\dagger} \hat{b}_{R} \hat{b}_{R}\right)$.

The Bose-Hubbard Hamiltonian describes the evolution of the many-particle system in the space restricted to two localized time-independent modes. The Bose-Hubbard time-dependent $N$-boson wavefunction takes on the form

$$
\left|\Psi_{B H}(t)\right\rangle=\sum_{n_{L}=0}^{N} C_{n_{L}}(t)\left|n_{L}, N-n_{L}\right\rangle,
$$

where the time-dependent coefficients $\left\{C_{n_{L}}(t)\right\}$ governing the evolution of the junction are simply obtained from the first-order equation

$$
\hat{H}_{B H}\left|\Psi_{B H}(t)\right\rangle=i\left|\dot{\Psi}_{B H}(t)\right\rangle .
$$

$\left\{\left|n_{L}, N-n_{L}\right\rangle\right\}$ are the Fock states assembled from the two 'modes' $\hat{b}_{L}^{\dagger}$ and $\hat{b}_{R}^{\dagger}$. Within the BH model the probability 
in the left well, Eq. (13), is conventionally identified with the occupation of the orbital $\phi_{L}$

$$
p_{L}(t)=\frac{1}{N}\left\langle\Psi_{B H}(t)\left|\hat{b}_{L}^{\dagger} \hat{b}_{L}\right| \Psi_{B H}(t)\right\rangle \text {. }
$$

The field operator contains only $M=2$ terms in the $\mathrm{BH}$ model and thus the fragmentation of the BEC reduces here to the occupation of the second natural orbital, $f=n_{2}^{(1)} / N \leq 50 \%$. Note that in the full manybody Schrödinger dynamics of bosonic Josephson junctions more than two natural orbitals can become occupied [8].

The dynamics governed by the Bose-Hubbard Hamiltonian occurs solely on the lowest band of the one-particle double-well problem. This emanates from Eq. (17), namely from retaining the first two terms only in the expansion of the field operator. It is in principle possible to retain more terms and include also higher band Wannier functions. This results in multi-band Bose-Hubbard models which approach the full many-particle Hamiltonian (6) with increasing number of included bands. However, in general many Wannier functions (Bloch bands) are needed to faithfully represent the full many-particle Hamiltonian (6) particularly when time-dependent phenomena are investigated.

\section{E. Multiconfigurational time-dependent Hartree for bosons (MCTDHB)}

In order to compute the time-evolution of the manybody Schrödinger equation we use the multiconfigurational time-dependent Hartree for bosons (MCTDHB) method, see Ref. [20] for theory and implementation and Refs. [8, 9, 24 26] for applications. In short, the MCTDHB method employs variational time-adaptive orbitals in the expansion of the many-boson wavefunction. With increasing number of orbitals the method converges to the exact many-body Schrödinger result [8, 9, 25], as was also shown by comparison to an exactly-solvable manybody model [26].

A faithful representation of the full many-body Hamiltonian (6) using time-independent orbitals often requires truncating the field operator (7) after a large number of terms. Even for few bosons this leads to prohibitively large Fock spaces. In the MCTDHB method time-dependent orbitals are used as opposed to the timeindependent ones in the expansion of the field operator

$$
\hat{\Psi}(x)=\sum_{j=1}^{M} \hat{b}_{j}(t) \phi_{j}(x, t)
$$

The orbitals are determined from the time-dependent variational principle. We briefly review the key features of the MCTDHB method.
Consider $M$ time-adaptive orbitals. When distributing $N$ bosons over the $M$ orbitals the resulting timedependent many-boson wavefunction reads:

$$
|\Psi(t)\rangle=\sum_{\vec{n}} C_{\vec{n}}(t)|\vec{n} ; t\rangle
$$

In Eq. (23) the $\left\{C_{\vec{n}}(t)\right\}$ are the time-dependent expansion coefficients and $\{|\vec{n} ; t\rangle\}$ the time-dependent permanents (symmetrized Hartree products) built from the $M$ time-dependent orbitals $\left\{\phi_{j}(x, t)\right\}$.

In the MCTDHB method the coefficients $\left\{C_{\vec{n}}(t)\right\}$ and the orbitals $\left\{\phi_{j}(x, t)\right\}$ of the many-boson wavefunction (23) are determined by the time-dependent variational principle. Explicitly, the MCTDHB equations of motion are derived by requiring stationarity of the many-body Schrödinger action functional

$$
\begin{aligned}
S\left[\left\{C_{\vec{n}}(t)\right\},\left\{\phi_{j}(x, t)\right\}\right] & =\int d t\left\{\left\langle\Psi(t)\left|\hat{H}-i \frac{\partial}{\partial t}\right| \Psi(t)\right\rangle\right. \\
& \left.-\sum_{k, j=1}^{M} \mu_{k j}(t)\left[\left\langle\phi_{k} \mid \phi_{j}\right\rangle-\delta_{k j}\right]\right\}
\end{aligned}
$$

with respect to variations of the time-dependent expansion coefficients $\left\{C_{\vec{n}}(t)\right\}$ and orbitals $\left\{\phi_{j}(x, t)\right\}$. Here, $\left\{\mu_{k j}(t)\right\}$ are time-dependent Lagrange multipliers which ensure the orthonormality of the orbitals throughout their propagation in time. The derivation is quite lengthy but otherwise straightforward, see [20] for details. Here we quote the final result for the MCTDHB equations of motion for the interaction potential $W\left(x-x^{\prime}\right)=$ $\lambda_{0} \delta\left(x-x^{\prime}\right)$ which read:

$$
i\left|\dot{\phi}_{j}\right\rangle=\hat{\mathbf{P}}\left[h\left|\phi_{j}\right\rangle+\lambda_{0} \sum_{k, s, l, q=1}^{M}\{\boldsymbol{\rho}(t)\}_{j k}^{-1} \rho_{k s l q} \phi_{s}^{*} \phi_{l}\left|\phi_{q}\right\rangle\right],
$$

with $j=1, \ldots, M$ for the time-dependent orbitals and

$$
\mathbf{H}(t) \mathbf{C}(t)=i \dot{\mathbf{C}}(t), \quad H_{\vec{n} \vec{n}^{\prime}}(t)=\left\langle\vec{n} ; t|\hat{H}| \vec{n}^{\prime} ; t\right\rangle
$$

for the expansion coefficients. Here, $\hat{\mathbf{P}}=1-$ $\sum_{u=1}^{M}\left|\phi_{u}\right\rangle\left\langle\phi_{u}\right|$ is a projector operator on the complementary space of the time-adaptive orbitals, $\boldsymbol{\rho}(t)$ the reduced one-particle density matrix, $\left\{\rho_{k s l q}\right\}$ the elements of the two-particle reduced density matrix, and $\mathbf{C}(t)=\left\{C_{\vec{n}}(t)\right\}$ the vector of expansion coefficients.

The use of $M$ optimized time-dependent orbitals leads to much faster numerical convergence to the full manybody Schrödinger results than an expansion in $M$ timeindependent orbitals. Thereby, problems involving large numbers of bosons can be solved on the full-many-body Schrödinger level. Indeed, numerical convergence to the exact time-dependent solution of the many-boson Schrödinger equation in closed [8] and open [23] trap potentials has been reported, as well as benchmarking against an exactly-solvable many-boson model [26]. For 
more details see [20]. The MCTDHB method has been cast as an efficient algorithm into a software package [21]. In practice, Eq. (26) is simplified by a mapping of the configuration space [22] which enables the efficient handling of millions of time-adaptive permanents [21].

\section{F. Gross-Pitaevskii mean-field}

One particular limiting case of the MCTDHB method deserves special attention: namely the case where only a single time-dependent orbital is used in the expansion of the field operator Eq. (22) and the many-body wavefunction Eq. (23), i.e. when $M=1$. In this case all particles occupy the same time-dependent orbital $\phi(x, t)$ at all times, i.e. there is only ever a single coefficient $C_{\vec{n}}$ and the solution of Eq. (26) becomes trivial. Thus, only Eq. (25) needs to be solved which differs from the well-known Gross-Pitaevskii mean-field equation

$$
i \dot{\phi}(x, t)=\left[h(x)+\lambda_{0}(N-1)|\phi(x, t)|^{2}\right] \phi(x, t) .
$$

only by an irrelevant overall phase. In other words, for $M=1$ the MCTDHB method reduces to GP mean-field theory. In GP theory the only free parameter is

$$
\lambda=\lambda_{0}(N-1)
$$

which is known as the mean-field interaction strength. In GP mean-field theory systems consisting of different numbers of particles, but with the same value of $\lambda$ lead to identical dynamics. Note that increasing $N$ at constant $\lambda$ implies a decreasing interaction strength $\lambda_{0}$.

By approximating the time-dependent GrossPitaevskii orbital as

$$
\phi(x, t)=a_{L}(t) \phi_{L}(x)+a_{R}(t) \phi_{R}(x)
$$

with $\left|a_{L}\right|^{2}+\left|a_{R}\right|^{2}=1$ a two-mode GP mean-field model can be derived [1, 2]. The model maps the dynamics of the BEC onto that of a classical nonrigid pendulum. Within the two-mode GP mean-field model the parameter

$$
\Lambda=\frac{U(N-1)}{2 J}
$$

plays an important role. Self-trapping, i.e. the inhibition of tunneling becomes possible if $\Lambda \geq \Lambda_{c}=2$ [1, 2]. The parameter $\Lambda$ is proportional to the mean-field interaction strength $\lambda$, see Eq. (5).

\section{RESULTS}

In this section we show that there is an unexpected universal dynamics on the full many-body Schrödinger level. We explain this universal many-body dynamics analytically and numerically using the $\mathrm{BH}$ model.

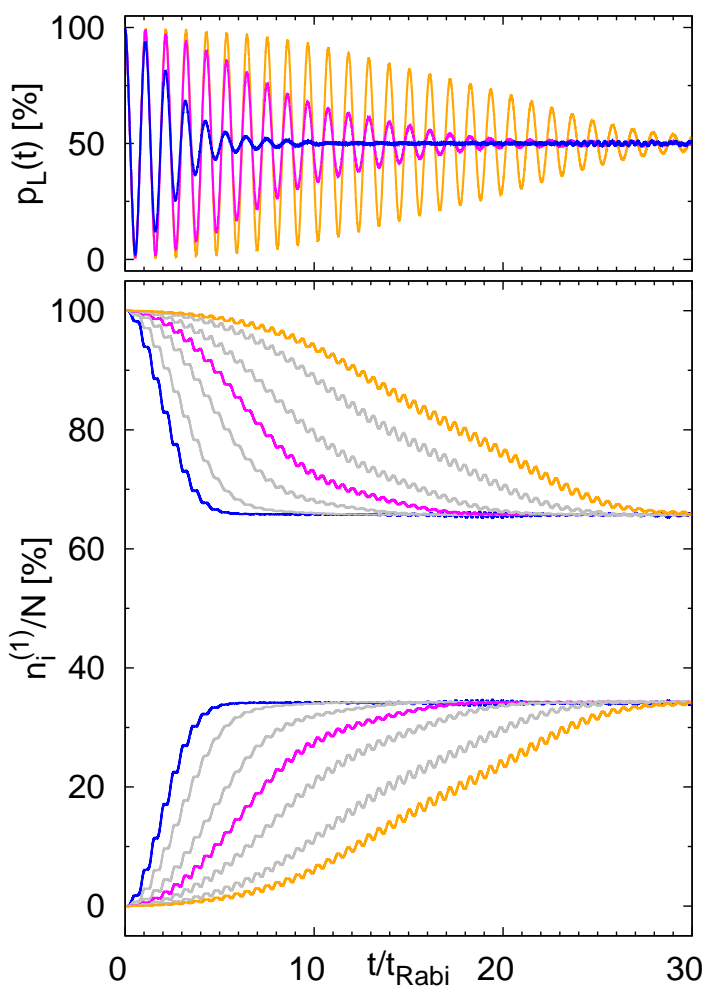

Figure 1. (color online): Universality of the many-body Schrödinger fragmentation dynamics. Top: shown is the probability in the left potential well $p_{L}(t)$ as a function of time for different numbers of particles. For all particle numbers the initial state is fully condensed, all bosons are in the left well $p_{L}(0)=1.0$, and the mean-field interaction strength is $\lambda=0.152(\Lambda=1.33)$. As the BECs tunnel back and forth through the barrier, the density oscillations collapse. Particle numbers: $\mathrm{N}=100$ (blue), 1000 (magenta), 10000 (orange). Bottom: shown are the corresponding natural occupations $n_{i}^{(1)} / N$ as a function of time. The grey lines represent particle numbers in between: $N=200,500,2000$ and 5000. Only two natural occupations $n_{1}^{(1)}$ and $n_{2}^{(1)}$ become significantly occupied in the dynamics. Thus, the fragmentation $f$ is given here by $f \approx n_{2}^{(1)} / N$, see Eq. (12). The fragmentation reaches a plateau at $f=f_{\text {col }}=34 \%$ after the collapse of the density oscillations for all particle numbers. The nature of the BEC changes from condensed to fragmented. All quantities shown are dimensionless.

\section{A. Many-body Schrödinger dynamics}

In the following we study the many-body Schrödinger dynamics in the bosonic Josephson junction for different particle numbers. We choose fully condensed initial states which are the GP ground states of the potential $V_{+}(x)$. Thus, initially the BECs are located in the left well. For each particle number $N$ the interaction strength $\lambda_{0}$ is chosen such that the mean-field interaction strength $\lambda$, defined in Eq. (28), is constant. We begin with $\lambda=0.152$ which corresponds to $\Lambda=1.33$, well below the critical value $\Lambda_{c}=2$ for self-trapping [1, 2]. 
Fig. 11(top) shows the probability in the left well $p_{L}(t)$ as a function of time for $N=100-10000$ bosons. The density tunnels back and forth through the potential barrier. However, the amplitude of the oscillations decreases and eventually the oscillations collapse. With increasing particle number the collapse occurs at later times. It is well known that the collapse is present in the many-body dynamics, but not within GP mean-field theory [1, 8]. We will therefore investigate the many-body nature of the BEC during the collapse in more detail.

In Fig. 11 (bottom) the corresponding natural occupations are shown. Since the many-body wavefunction is initially condensed, there is only one natural occupation $n_{1}^{(1)}=N$ at $t=0$ and the fragmentation $f$ is zero, see Eq. (12). However, as the condensate tunnels back and forth through the barrier a second natural orbital becomes occupied. The nature of the BEC changes from condensed to fragmented as the density oscillations collapse. We stress that these results represent the manybody Schrödinger dynamics of the Hamiltonian (11).

For the smallest particle number, $N=100$ bosons, $M=4$ orbitals were used in the MCTDHB computation and the result is numerically exact. However, only $M=2$ time-dependent variationally optimized orbitals are needed here: there is only a small quantitative difference between computations with $M=4$ and $M=2$ orbitals. Until time $t=30 t_{R a b i}$ the occupations of the third and fourth natural orbitals together stay below $0.1 \%$ and are not shown in Fig. 1. Therefore, $M=2$ orbitals were used in all computations for larger particle numbers.

From Fig. 1 (bottom) we see that the fragmentation dynamics is universal in the following sense: at constant mean-field interaction strength $\lambda$ systems consisting of different numbers of bosons fragment to the same value during the collapse of the density oscillations. The two largest natural occupations reach plateaus at about $n_{1}^{(1)} / N=66 \%$ and $n_{2}^{(1)} / N=34 \%$. Using Eq. (12) we find for the fragmentation after the collapse of the density oscillations $f=34 \%$ regardless of the number of particles involved. From now on we refer to the fragmentation after the collapse of the density oscillations as $f_{\mathrm{col}}$.

This universal behavior is fundamentally different from that within GP theory, where the dynamics is strictly identical for all systems with the same value of $\lambda$, regardless of the number of particles, see Sec. IIF. The GP mean-field approximation excludes any possibility for fragmentation. Here, we report on a universal fragmentation dynamics which occurs naturally on the many-body level, i.e., without being enforced by an approximation. Fragmentation of condensates is usually strongly dependent on the number of particles in the system [8, 13 16], see also the discussion of Ref. [32 in Sec. IIB]in this context. Hence, the appearance of a universal fragmentation dynamics is highly unexpected.

We note that the times until a given fragmentation is reached increases only logarithmically with the number of particles, implying that the phenomenon occurs also for large particle numbers. Details on this logarithmic

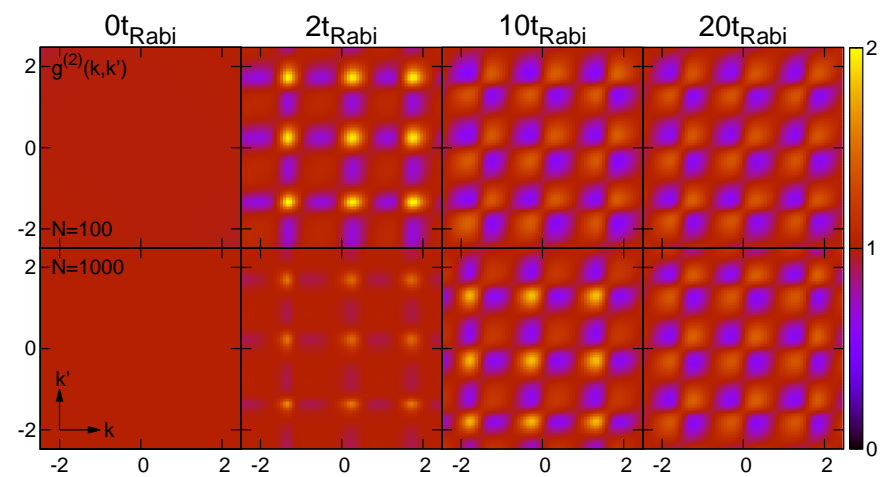

Figure 2. (color online): Universality of the many-body Schrödinger two-particle momentum correlations. Shown is the momentum correlation function $g^{(2)}\left(k, k^{\prime}\right)$ at different times for two of the cases shown in Fig. 1] Top row: $N=100$ bosons. Initially the system is condensed and there are no correlations (leftmost panel). During the collapse of the density oscillations correlations build up and the momentum correlation function varies rapidly in time (second panel). For $t \geq 7 t_{\text {Rabi }}$ the density oscillations have collapsed, see Fig. 1 (top). The structure of $g^{(2)}\left(k, k^{\prime}\right)$ remains practically constant in time then (third and fourth panel). Bottom Row: Same as top row, but for $N=1000$ bosons. The collapse takes until about $t=20 t_{\text {Rabi }}$ for this larger particle number and the structure of $g^{(2)}\left(k, k^{\prime}\right)$ becomes constant from then on (fourth panel). The values of $g^{(2)}\left(k, k^{\prime}\right)$ after the collapse are the same for both particle numbers and show how the universality of fragmentation leads to the universality of directly observable quantities. All quantities shown are dimensionless.

scaling are given in appendix A

In order to establish how the universality of fragmentation can be observed, we focus on the momentum correlation function $g^{(2)}\left(k, k^{\prime}\right)$. Fig. 2 shows $g^{(2)}\left(k, k^{\prime}\right)$ at different times for $N=100$ and $N=1000$ bosons. The initial state is condensed $n_{1}^{(1)}=N$ and therefore $g^{(2)}\left(k, k^{\prime}\right)=1-1 / N$, i.e. $g^{(2)}\left(k, k^{\prime}\right)$ is completely flat. As the system starts to fragment, correlations between particles build up and $g^{(2)}\left(k, k^{\prime}\right)$ exhibits a time-varying structure. However, once the density oscillations have collapsed $g^{(2)}\left(k, k^{\prime}\right)$ is nearly constant in time. It is clearly seen from the rightmost panels that the momentum correlation functions for $N=100$ and $N=1000$ bosons are the same then. This is the case for all particle numbers considered in this work. Thus, the universality of fragmentation manifests itself unambiguously as a measurable universality of momentum correlations.

The periodic spacing of $g^{(2)}\left(k, k^{\prime}\right)$ in the rightmost panels is determined by the distance $d=4$ between the two wells of the double well potential. This becomes clear by considering an analytical model for a fragmented condensate consisting of two fragments of $N / 2$ bosons located in the left and right wells with equal occupations 
$n_{1}^{(1)} / N=n_{2}^{(1)} / N=50 \%$. For such a condensate one finds $g^{(2)}\left(k, k^{\prime}\right) \propto 1+N /(N-1) \cos \left[\left(k-k^{\prime}\right) d\right] / 2$, see Ref. [27], i.e. $g^{(2)}\left(k, k^{\prime}\right)$ becomes periodic in $k$ and $k^{\prime}$ with period $2 \pi / d$. The case shown in Fig. 2 is more complicated and lies in between the extremes of a fully condensed state with a flat $g^{(2)}\left(k, k^{\prime}\right)$ and the fragmented model state described above. However, the periodicity of $g^{(2)}\left(k, k^{\prime}\right)$ in $k$ and $k^{\prime}$ with period $\pi / 2$ is already clearly visible.

\section{B. Bose-Hubbard dynamics}

So far we have established the universality of fragmentation of the many-body Schrödinger dynamics. We will now use a simple model to show that the universality of fragmentation is a general many-body phenomenon that exists for a wide range of initial conditions and explain its origin. As a model we use the two-mode BH Hamiltonian Eq. (18). by

We consider the family of condensed initial states given

$$
\left|\Psi_{0}\right\rangle=\frac{1}{\sqrt{N !}}\left(\sqrt{p_{L}(0)} b_{L}^{\dagger}+\sqrt{p_{R}(0)} b_{R}^{\dagger}\right)^{N}|0\rangle
$$

and begin by choosing parameter values corresponding to the case considered in the previous section for the manybody Schrödinger dynamics, $\lambda=0.152(\Lambda=1.33)$. For the initial states Eq. (31) we solve the dynamics using the BH Hamiltonian (18). We find that for the entire family of initial states $\left|\Psi_{0}\right\rangle$ with $0 \leq p_{L}(0) \leq 1$, BECs consisting of different numbers of particles fragment to the same value $f_{c o l}$ during the collapse of the density oscillations. Thus, also the BH fragmentation dynamics is universal. This can be seen in Fig. 3 (left) where the BH fragmentation is shown as a function of time for $N=1000$ and 10000 bosons. The fragmentation of initial states with $p_{L}(0)=1.0$ reaches a plateau at about $f_{c o l}=32 \%$, not far from the many-body Schrödinger result 34\%, see Fig. 1 (bottom). Similarly, initial states with $p_{L}(0)=0.8$ fragment towards the value $f_{c o l}=16 \%$. Thus, the universality of fragmentation is a general phenomenon which exists for a whole family of initially-condensed systems.

Since fragmentation is a phenomenon that occurs due to interparticle interactions, we now investigate whether the universality of fragmentation is robust with respect to reducing the interaction strength. For this purpose we consider an interaction strength that is ten times smaller, $\lambda=0.0152(\Lambda=0.133)$. The results for the fragmentation as a function of time are shown in Fig. 4 (left). Analogous to Fig. 3 the dynamics is shown for the two condensed initial states with $p_{L}(0)=1.0$ and $p_{L}(0)=0.8$.

As in the case for stronger interaction we find that BECs with different particle numbers fragment to the same value in the course of time. Condensed initial states with all particles in the left well, $p_{L}(0)=1.0$, now fragment to about $f_{c o l}=48.3 \%$ which is higher than for stronger interaction. Condensed initial states with $p_{L}(0)=0.8$ fragment to about $f_{\text {col }}=10.6 \%$ which is

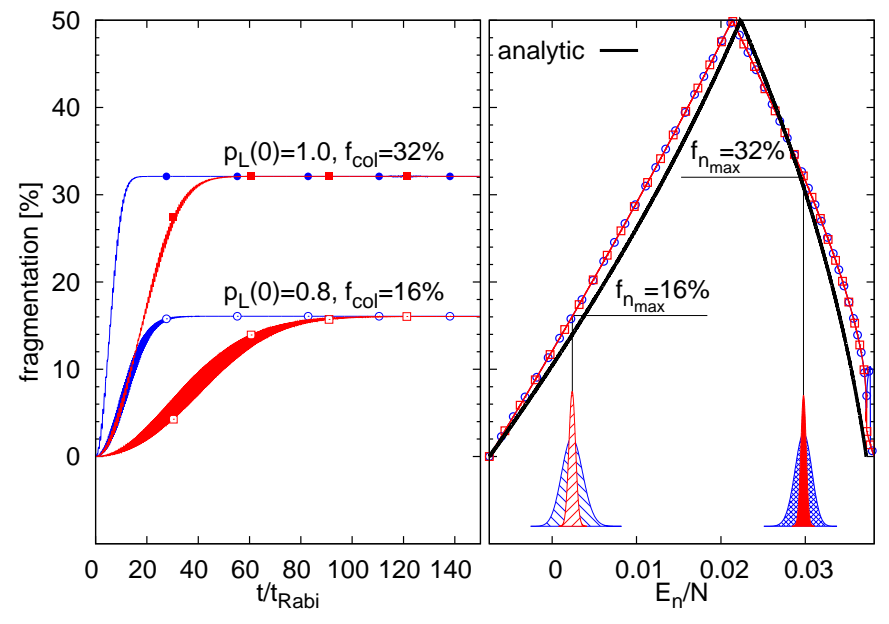

Figure 3. (color online): Universality of the Bose-Hubbard fragmentation dynamics. Left panel: Shown is the fragmentation $f$ as a function of time for different condensed initial states and for different numbers of particles. The initial states $\left|\Psi_{0}\right\rangle$ are defined in Eq. (31). The mean-field interaction strength is the same as in Fig. 1 for all particle numbers. For initial states with $p_{L}(0)=1.0$ the $\mathrm{BH}$ fragmentation reaches a plateau after the collapse of the density oscillations at $f=f_{\text {col }}=32 \%$, not far from the many-body Schrödinger result, see Fig. 1. The approximative formula for $\Lambda \ll 1$, Eq. (47), predicts $f_{\text {col }}=1 / 2-\Lambda / 8=33.3 \%$, which is also very close. Similarly, for condensed initial states with $p_{L}(0)=0.8$ a plateau is reached at $f=f_{\text {col }}=16 \%$. Parameter values: $N=1000, p_{L}(0)=1.0: \bullet ; N=1000, p_{L}(0)=0.8: \odot ;$ $N=10000, p_{L}(0)=1.0: \square ; N=10000, p_{L}(0)=0.8: \square$. Right panel: for each eigenstate $\left|E_{n}\right\rangle$ of the $\mathrm{BH}$ model its fragmentation $f_{n}$ is shown as a function of the eigenstate energy per particle $E_{n} / N$. Curves for different particle numbers lie on top of each other $(\mathrm{N}=1000$ : $\odot ; \mathrm{N}=10000$ : $\square)$. The shaded areas underneath represent the distributions of coefficients $C_{n}=\left\langle E_{n} \mid \Psi_{0}\right\rangle$ of the initial states discussed above $\left(\mathrm{N}=1000\right.$ : blue; $\mathrm{N}=10000$ : $\mathrm{red} ; p_{L}(0)=0.8$ : distributions on the left ; $p_{L}(0)=1.0$ : distributions on the right). The distributions are sharply peaked around the index $n=n_{\max }$ for which the eigenstate energy $E_{n}$ equals the energy of the initial state $E$ (vertical lines). The corresponding eigenstate fragmentation $f_{n_{\max }}$ is indicated as well. The analytical result (solid black line) for the eigenstate fragmentation $f_{n}$ as function of the eigenstate energy $E_{n}$ is also shown and approximates the numerical one well. Comparing the left and right panel it is seen that $f_{\text {col }}=f_{n_{\max }}$. This connection between the initial state and the fragmentation after the collapse of the density oscillations holds numerically for the entire family of initial states $\left|\Psi_{0}\right\rangle$ and is predicted analytically by Eq. (46). See text for details. All quantities shown are dimensionless.

lower than for stronger interaction. Note that the time scale is now roughly ten times larger for this interaction strength. Nevertheless, even for large particle numbers the BECs eventually fragment to the same value. Thus, the $\mathrm{BH}$ dynamics is universal and many-body in nature over a wide range of interaction strengths. 


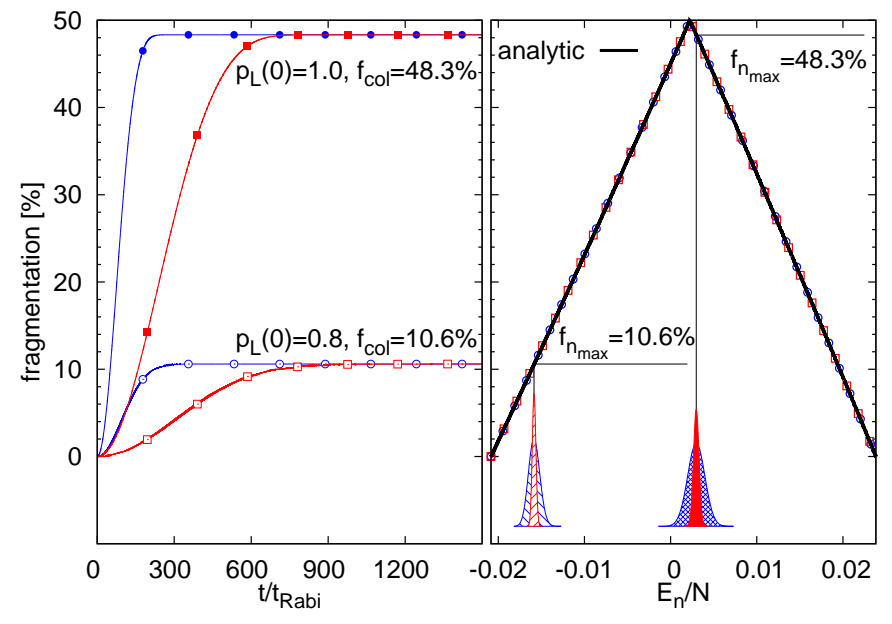

Figure 4. (color online). Universality of the Bose-Hubbard fragmentation dynamics for weaker interaction. The meanfield interaction strength is now ten-times smaller, $\lambda=0.0152$ $(\Lambda=0.133)$. Left panel: as in Fig. 3 The fragmentation after the collapse of the density oscillations is now $f=f_{\text {col }}=48.3 \%$ for initial states with $p_{L}(0)=1.0$. Here, the approximative formula for $\Lambda \ll 1$, Eq. (47), predicts $f_{\text {col }}=1 / 2-\Lambda / 8=48.3 \%$ and agrees perfectly with the data. For initial states with $p_{L}(0)=0.8$ one finds $f=f_{\text {col }}=10.6 \%$. These values are, respectively, higher and lower than for stronger interaction. Note the different, slower time scale compared to the case of stronger interaction. Right panel: as in Fig. 3. The analytical result (solid black line) for the eigenstate fragmentation $f_{n}$ as function of the eigenstate energy $E_{n}$ provides an excellent approximation to the numerical one here. By comparing the left and right panels one again finds numerically that $f_{\text {col }}=f_{n_{\max }}$ for the entire family of condensed initial states $\left|\Psi_{0}\right\rangle$, as predicted analytically by Eq. (46). All quantities shown are dimensionless.

\section{Analytical prediction of the universality of fragmentation}

In this section we provide an analytical explanation of the universality of fragmentation based on the $\mathrm{BH}$ model. To this end we calculate approximatively the elements of the $\mathrm{BH}$ first-order reduced-density matrix, Eq. (10), after the collapse of the density oscillations, diagonalize the matrix to obtain the eigenvalues $n_{i}^{(1)}$ and calculate the fragmentation.

We consider the family of initial states given by Eq. (31). The energy of the initial state $E=\left\langle\Psi_{0}\left|H_{B H}\right| \Psi_{0}\right\rangle$ can be evaluated and yields

$E=-2 N J \sqrt{p_{L}(0) p_{R}(0)}+\frac{U N(N-1)}{2}\left(p_{L}(0)^{2}+p_{R}(0)^{2}\right)$.

Next, we consider the spectrum of the BH Hamiltonian

$$
\hat{H}_{B H}\left|E_{n}\right\rangle=E_{n}\left|E_{n}\right\rangle \text {. }
$$

and note that all eigenstates are parity eigenstates $\hat{P}\left|E_{n}\right\rangle= \pm\left|E_{n}\right\rangle$ because the potential $V(x)$ is symmet- ric. The time-dependent $\mathrm{BH}$ wave function $|\Psi(t)\rangle$ can be expanded in the $\mathrm{BH}$ eigenstates $\left|E_{n}\right\rangle$

$$
|\Psi(t)\rangle=\sum_{m=0}^{N} C_{n}\left|E_{n}\right\rangle e^{-i E_{n} t}
$$

with coefficients $C_{n}=\left\langle E_{n} \mid \Psi(0)\right\rangle$. By treating the interaction as a perturbation to the noninteracting system the first-order perturbative expression for the BH energy eigenvalues reads

$$
E_{n}=-J(N-2 n)+\frac{U}{2}(N-n) n+\frac{U N(N-1)}{4} .
$$

For a noninteracting system the coefficients $C_{n}$ are binomially distributed with a width $\sim 1 / \sqrt{N}$, i.e. the distribution becomes sharply peaked for $N \gg 1$. The energy levels are equidistantly spaced $E_{n+1}-E_{n}=2 J$. Thus, in the noninteracting case the system of bosons always returns precisely to its initial state after a time interval of $\pi / J=t_{\text {Rabi }}$, regardless of its initial state. However, a nonzero interaction modifies the energy spectrum, shifts the distribution of the $C_{n}$ and causes the contributions from different eigenstates to dephase in time. This leads eventually to the collapse of the density oscillations.

Assuming that the distribution of coefficients $C_{n}$ also remains sharply peaked around a value $n_{\max }$ in the interacting case, the location of the maximum of the distribution can be determined by requiring that the energy of the initial state equals the energy of a $\mathrm{BH}$ eigenstate. By equating Eqs. (35) and (32) one finds

$$
\begin{aligned}
& \frac{n_{\max }}{N}=\frac{1}{2}+\frac{1}{\Lambda}- \\
& \sqrt{\frac{1}{\Lambda^{2}}+\frac{2 \sqrt{p_{L}(0) p_{R}(0)}}{\Lambda}+\frac{3}{4}-\left(p_{L}(0)^{2}+p_{R}(0)^{2}\right)}
\end{aligned}
$$

in the limit where $N-1 \approx N$. Using $\hat{b}_{L, R}=\frac{1}{\sqrt{2}}\left(\hat{b}_{g} \pm \hat{b}_{u}\right)$ and $\hat{n}_{i}=\hat{b}_{i}^{\dagger} \hat{b}_{i}$ the elements of the first-order reduced density matrix read

$$
\begin{aligned}
\rho_{L L}(t) & =\frac{1}{2}\left\langle\Psi(t)\left|\hat{n}_{g}+\hat{n}_{u}+\hat{b}_{u}^{\dagger} \hat{b}_{g}+\hat{b}_{g}^{\dagger} \hat{b}_{u}\right| \Psi(t)\right\rangle \\
\rho_{R R}(t) & =\frac{1}{2}\left\langle\Psi(t)\left|\hat{n}_{g}+\hat{n}_{u}-\hat{b}_{u}^{\dagger} \hat{b}_{g}-\hat{b}_{g}^{\dagger} \hat{b}_{u}\right| \Psi(t)\right\rangle \\
\rho_{L R}(t) & =\frac{1}{2}\left\langle\Psi(t)\left|\hat{n}_{g}-\hat{n}_{u}+\hat{b}_{u}^{\dagger} \hat{b}_{g}-\hat{b}_{g}^{\dagger} \hat{b}_{u}\right| \Psi(t)\right\rangle .
\end{aligned}
$$

Assuming that oscillatory terms average to zero and using the fact that due to symmetry $\left\langle E_{n}\left|\hat{b}_{u}^{\dagger} \hat{b}_{g}\right| E_{n}\right\rangle=$ $\left\langle E_{n}\left|\hat{P}^{\dagger} \hat{P} \hat{b}_{u}^{\dagger} \hat{b}_{g} \hat{P}^{\dagger} \hat{P}\right| E_{n}\right\rangle=-\left\langle E_{n}\left|\hat{b}_{u}^{\dagger} \hat{b}_{g}\right| E_{n}\right\rangle=0$ one finds

$$
\begin{aligned}
& \left\langle\Psi(t)\left|\hat{b}_{u}^{\dagger} \hat{b}_{g}\right| \Psi(t)\right\rangle= \\
& \sum_{m \neq n} C_{m}^{*} C_{n}\left\langle E_{m}\left|\hat{b}_{u}^{\dagger} \hat{b}_{g}\right| E_{n}\right\rangle e^{i\left(E_{m}-E_{n}\right) t}=0,
\end{aligned}
$$


and similarly

$$
\begin{aligned}
& \left\langle\Psi(t)\left|\hat{n}_{u}\right| \Psi(t)\right\rangle \\
& =\sum_{m, n} C_{m}^{*} C_{n}\left\langle E_{m}\left|\hat{n}_{u}\right| E_{n}\right\rangle e^{i\left(E_{m}-E_{n}\right) t} \\
& =\sum_{n}\left|C_{n}\right|^{2}\left\langle E_{n}\left|\hat{n}_{u}\right| E_{n}\right\rangle \approx n_{\max }
\end{aligned}
$$

where in the last approximation it was used that the coefficient distribution is sharply peaked at $n=n_{\max }$. Substituting Eqs. (40) and (41) into Eqs. (37), (38) and (39) and using $N=n_{u}+n_{g}$ one arrives at

$$
\begin{gathered}
\rho_{L L}=\rho_{R R}=\frac{N}{2} \\
\rho_{L R}=\frac{N}{2}-n_{\max } .
\end{gathered}
$$

By diagonalizing the matrix $\rho_{i j}$ one finds $n_{1,2}^{(1)}=$ $n_{\max }, N-n_{\max }$ and thus using Eq. (12)

$$
f_{\text {col }}=\min \left[\frac{n_{\max }}{N}, 1-\frac{n_{\max }}{N}\right] .
$$

The universality of fragmentation for different particle numbers now follows directly from Eq. (36): the values for $n_{\max } / N$ and therefore for $f_{\text {col }}$ depend on $N$ only through the parameter $\Lambda$ and thus all systems with the same value of $\Lambda$ fragment to the same value $f_{\text {col }}$, regardless of the number of particles. We also note that the fragmentation of each eigenstate $\left|E_{n}\right\rangle$ is given to lowest order by

$$
f_{n}=\min \left[\frac{n}{N}, 1-\frac{n}{N}\right] .
$$

Therefore the fragmentation after the collapse of the density oscillations $f_{\text {col }}$ given in Eq. (44) is the same as that of the eigenstate around which the distribution of coefficients $C_{n}$ is peaked, and thus

$$
f_{\text {col }}=f_{n_{\text {max }}} .
$$

It is instructive to expand Eq. (36) for two limiting cases. If initially all particles are in one of the wells, $p_{L}(0)=1$ or $p_{L}(0)=0$, and $\Lambda \ll 1$ Eq. (44) reduces to

$$
f_{\text {col }}=\frac{1}{2}-\frac{\Lambda}{8},
$$

predicting strong fragmentation at arbitrarily weak interaction strengths and for arbitrarily large numbers of particles after the collapse of the density oscillations.

In contrast, for the initial state with equal numbers of particles in both wells, $p_{L}(0)=p_{R}(0)=1 / 2$, Eq. (36) predicts $n_{\max }=0$ and according to Eq. (44)

$$
f_{\text {col }}=0,
$$

indicating that no fragmentation should occur. Thus, whether or not the system of bosons fragments is expected to depend strongly on the initial state.

\section{Numerical verification}

In this section we test the analytical predictions of the previous section. To this end we diagonalize the BH Hamiltonian, Eq. (18), and evaluate the respective quantities. The right panel of Fig. 3 shows the fragmentation $f_{n}$ of each $\mathrm{BH}$ eigenstate as a function of its energy per particle for $N=1000$ and $N=10000$ bosons. The curves for different particle numbers lie on top of each other. Also shown are the sharply peaked distributions of the expansion coefficients $C_{n}$ for the initial states with $p_{L}(0)=0.8$ and $p_{L}(0)=1.0$. The values of the eigenstate fragmentations $f_{n}$ at the peaks of the distributions are shown as well. The solid black line represents the approximative analytical result for the eigenstate fragmentations as a function of the perturbative energy per particle, i.e. $f_{n}$ given by Eq. (45) as a function of $E_{n} / N$ with $E_{n}$ given by Eq. (35). The analytic result provides good approximation to the numerical one at this interaction strength. By comparing the left and the right panels of Fig. 3 it is clearly seen that $f_{c o l}=f_{n_{\max }}$, as expected on the basis of Eq. (46).

The same is true for the case of ten-times weaker interaction strength, shown in Fig. 4. Comparing the analytical predictions in Figs. 3 and 4 it is clearly seen that for weaker interaction strength the approximative analytical results improve.

Lastly, we check the predictions of Eqs. (47) and (48). For the initial states with $p_{L}(0)=1$ Eq. (47) predicts a fragmentation after the collapse of $f_{\text {col }}=33.3 \%(48.3 \%)$ for $\Lambda=1.33(0.133)$ in good agreement with the numerical values. We note that for the initial state with $p_{L}(0)=0.5$ there are no density oscillations between the two wells and thus there can also be no collapse. Nevertheless, in agreement with Eq. (48) no appreciable fragmentation occurs in the dynamics: numerically, one finds that the fragmentation of the $\mathrm{BH}$ model oscillates between $0 \%$ and $0.2 \%$.

These results show that the fragmentation after the collapse of the density oscillations $f_{c o l}$ depends crucially on the initial state: initial states with large differences in particle numbers between the left and the right well fragment strongly, even if $\Lambda \ll 1$, i.e. if the mean-field interaction strength $\lambda$ is very small. Therefore, there is no weakly interacting limit where the GP mean-field is valid at long times. On the other hand, condensed initial states with similar numbers of particles in both wells do not fragment strongly in time.

\section{CONCLUSIONS}

Summarizing, we have shown that there is a universal fragmentation dynamics in bosonic Josephson junctions at weak interaction strengths by solving the timedependent many-boson Schrödinger equation. The phenomenon can be detected, for instance, through a measurement of the two-particle momentum correlation func- 


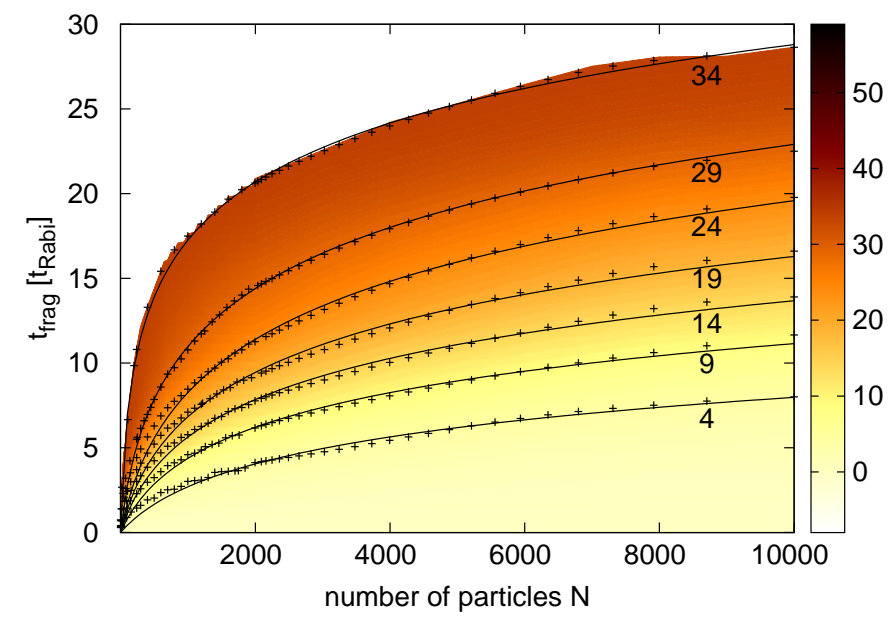

Figure 5. (color online): Many-body Schrödinger fragmentation times. Shown are the times $t_{f r a g}$ that are needed to reach a given fragmentation $f$ as a function of the number of bosons for the same parameters as in Fig. 1. The fragmentation values (crosses) are well described by a logarithmic fit function (lines), here shown for the values $f=4 \%, 9 \%, \ldots, 34 \%$. This logarithmic dependence of $t_{\text {frag }}$ on the number of particles implies the breakdown of GP mean-field theory even in the limit of large particle numbers. See text for details. All quantities shown are dimensionless.

tions. We have shown that the two-mode Bose-Hubbard model approximates the many-body Schrödinger results quite well here. The origin of the universal fragmentation dynamics can be explained analytically and numerically using the two-mode Bose-Hubbard model. We found that the extent to which many-body effects become important at later times depends crucially on the initial state. The value of the fragmentation of initially condensed states at later times can be precisely predicted analytically in the limit of weak interactions. At arbitrarily weak interaction strength and for arbitrarily large numbers of particles there are condensed initial states that fragment strongly. Thus, there is no weakly interacting limit where the Gross-Pitaevskii mean-field is valid at long times.

\section{ACKNOWLEDGMENTS}

Financial support by the DFG is gratefully acknowledged as is computing time at the High Performance Computing Center Stuttgart (HLRS). K.S. acknowledges funding through the Karel Urbanek Postdoctoral Research Fellowship. Discussions with Mark Kasevich are gratefully acknowledged.

\section{Appendix A: Logarithmic scaling of the fragmentation time}

Here we briefly discuss the time scales involved in the Schrödinger fragmentation dynamics shown in Fig. 1] We define the fragmentation time $t_{\text {frag }}$ as the first time at which a certain fragmentation $f$ is reached. Fig. 5 shows $t_{\text {frag }}$ as a function of $N$. Clearly, $t_{\text {frag }}$ increases with $N$. For any value of $f, t_{\text {frag }}$ is well described by a fit to the function $t(N)=a \ln (1+b N)$, as is shown here for the values $f=4 \%, 9 \%, \ldots, 34 \%$. Thus, $t_{\text {frag }}$ grows logarithmically with $N$. Consequently, the fragmentation does not decrease or even disappear in the limit of large $N$. Even for $N=10000$ bosons the fragmentation rises to about $10 \%$ in less than a dozen $t_{\text {Rabi }}$. As GP theory does not allow BECs to fragment at all, $t_{\text {frag }}$ defines a measure for the breakdown of mean-field theory. Thus, we find here a logarithmic dependence of the mean-field theory breakdown time on $N$ based on the time-dependent many-body Schrödinger dynamics. We note that a logarithmic breakdown of mean-field theory has been reported earlier based on the $\mathrm{BH}$ model [33].
[1] G. J. Milburn, J. Corney, E. M. Wright, D. F. Walls, Phys. Rev. A 55, 4318 (1997).

[2] A. Smerzi, S. Fantoni, S. Giovanazzi, S. R. Shenoy, Phys. Rev. Lett. 79, 4950 (1997).

[3] M. Albiez, R. Gati, J. Fölling, S. Hunsmann, M. Cristiani, M. K. Oberthaler, Phys. Rev. Lett. 95, 010402 (2005).

[4] S. Levy, E. Lahoud, I. Shomroni, J. Steinhauer, Nature (London), 449, 579 (2007).

[5] T. Zibold, E. Nicklas, C. Gross, and M. K. Oberthaler, Phys. Rev. Lett. 105, 204101 (2010).

[6] C. Orzel, A. K. Tuchman, M. L. Fenselau, M. Yasuda, and M. A. Kasevich, Science 291, 2386 (2001).

[7] T. Schumm, S. Hofferberth, L. M. Andersson, S. Wildermuth, S. Groth, I. Bar-Joseph, J. Schmiedmayer, and P. Krüger, Nature Physics 1, 57 (2005).

[8] K. Sakmann, A. I. Streltsov, O. E. Alon, and L. S. Cederbaum, Phys. Rev. Lett. 103, 220601 (2009).
[9] K. Sakmann, A. I. Streltsov, O. E. Alon, and L. S. Cederbaum, Phys. Rev. A 82, 013620 (2010).

[10] P. Nozières, D. Saint James, J. Phys. (France) 43, 1133 (1982); P. Nozières, in Bose-Einstein Condensation, edited by A. Griffin, D. W. Snoke, and S. Stringari (Cambridge University Press, Cambridge, England, 1996).

[11] A. J. Leggett, Quantum Liquids (Oxford University Press, 2006), p. 31.

[12] O. Penrose and L. Onsager, Phys. Rev. 104, 576 (1956).

[13] R. W. Spekkens and J. E. Sipe, Phys. Rev. A 59, 3868 (1999).

[14] A. I. Streltsov, O. E. Alon, and L. S. Cederbaum, Phys. Rev. A 73, 063626 (2006).

[15] E. J. Mueller, T.-L. Ho, M. Ueda, and G. Baym, Phys. Rev. A 74, 033612 (2006).

[16] P. Bader and U. R. Fischer, Phys. Rev. Lett. 103, 060402 (2009). 
[17] S. Hofferberth, I. Lesanovsky, B. Fischer, J. Verdu, and J. Schmiedmayer, Nature Physics 2, 710 (2006).

[18] S. Raghavan, A. Smerzi, and V. M. Kenkre, Phys. Rev. A 60, R1787 (1999).

[19] A. Vardi and J. R. Anglin, Phys. Rev. Lett. 86, 568 (2001).

[20] A. I. Streltsov, O. E. Alon, and L. S. Cederbaum, Phys. Rev. Lett. 99, 030402 (2007); O. E. Alon, A. I. Streltsov, and L. S. Cederbaum, Phys. Rev. A 77, 033613 (2008); http://MCTDHB.org.

[21] A. I. Streltsov, K. Sakmann, A. U. J. Lode, O. E. Alon, and L. S. Cederbaum, The Multiconfigurational TimeDependent Hartree for Bosons Package, version 2.1, Heidelberg (2011).

[22] A. I. Streltsov, O. E. Alon, and L. S. Cederbaum, Phys. Rev. A 81, 022124 (2010).

[23] A. U. J. Lode, A. I. Streltsov, K. Sakmann, O. E. Alon, and L. S. Cederbaum, Proc. Natl. Acad. Sci. USA 109, 13521 (2012).

[24] K. Sakmann, A. I. Streltsov, O. E. Alon, and L. S. Cederbaum, New J. Phys. 13, 043003 (2011).
[25] A. I. Streltsov, K. Sakmann, O. E. Alon, and L. S. Cederbaum, Phys. Rev. A 83, 043604 (2011).

[26] A. U. J. Lode, K. Sakmann, O. E. Alon, L. S. Cederbaum, and A. I. Streltsov, Phys. Rev. A 86, 063606 (2012).

[27] K. Sakmann, A. I. Streltsov, O. E. Alon, and L. S. Cederbaum, Phys. Rev. A 78, 023615 (2008).

[28] M. Naraschewski and R. J. Glauber, Phys. Rev. A 59, 4595 (1999).

[29] S. Fölling, F. Gerbier, A. Widera, O. Mandel, T. Gericke, and I. Bloch, Nature (London), 434, 481 (2005).

[30] A. Perrin, H. Chang, V. Krachmalnicoff, M. Schellekens, D. Boiron, A. Aspect, and C. I. Westbrook, Phys. Rev. Lett. 99, 150405 (2007).

[31] A. Perrin, R. Bücker, S. Manz, T. Betz, C. Koller, T. Plisson, T. Schumm, and J. Schmiedmayer, Nature Physics 8, 195 (2012).

[32] E. H. Lieb and R. Seiringer, Phys. Rev. Lett. 88, 170409 (2002).

[33] A. Vardi and J. R. Anglin, Phys. Rev. Lett. 86, 568 (2001). 